\title{
Molecular Dynamics of SARS-CoV-2 Nsp1 Structural Changes Associated with Variant Mutations
}

\section{Shokouh Rezaei}

Shahid Beheshti University

Yahya Sefidbakht ( $\nabla$ y_sefidbakht@sbu.ac.ir)

Shahid Beheshti University https://orcid.org/0000-0003-0538-0252

\section{Filipe Pereira}

University of Coimbra: Universidade de Coimbra

\section{Research Article}

Keywords: SARS-CoV-2, Nsp1, Ribosome, Mutations, Molecular dynamics.

Posted Date: August 12th, 2021

DOI: https://doi.org/10.21203/rs.3.rs-781636/v1

License: (c) (1) This work is licensed under a Creative Commons Attribution 4.0 International License. Read Full License 


\section{Abstract}

SARS-CoV-2 non-structural protein 1 (Nsp1) is a virulence factor that inhibits the translation of host mRNAs and interact with viral RNA. Despite the relevance of Nsp1, few studies have been conducted to understand the effect of mutations on Nsp1 structure and function. Here, we provide a molecular dynamics simulation of SARS-CoV-2 Nsp1, wild type and variants. We found that SARS-CoV-2 Nsp1 has a more $\mathrm{R}_{\mathrm{g}}$ value than SARS-CoV-1 Nsp1, with indicate an effect on the folding protein. This result suggest that SARS-CoV-2 Nsp1 can more easily approach the active site of the ribosome compared to SARS-CoV1 Nsp1. In addition, we found that the C-terminal of the SARS-CoV-2 Nsp1, in particular residues 164 to 170, are more flexible than other regions of SARS-CoV-2 Nsp1 and SARS-CoV-1 Nsp1, confirming the role of this region in the interaction with the $40 \mathrm{~S}$ subunit. Moreover, multiple deletion mutations have been found in the N/C-terminal of the SARS-CoV-2 Nsp1, which seems the effect of SARS-CoV-2 Nsp1 multiple deletions is greater than that of substitutions. Among all deletions, D156-158 and D80-90 may destabilize the protein structure and possibly increase the virulence of the SARS-CoV-2. Overall, our findings reinforce the importance of studying Nsp1 conformational changes in new variants and its effect on virulence of SARS-CoV-2.

\section{Introduction}

The 5'-end of SARS-CoV-2 genome includes more than two-thirds of RNA genome that comprises two large open reading frames (ORFs/ORF1ab) [1]. ORF1ab proteolytically processed by two virally encoded proteinases (PLpro and $\mathrm{M}^{\mathrm{pro}}$ ) to produce16 non-structural proteins (NSP1 to NSP16) [2][3]. Non-structure protein 1 (Nsp1) is encoded by the gene closest to the 5 '-end of the SARS-CoV-2 genome, being also known as leader protein $[4,5]$. NSP1 has identical physical features and biological activity with other $\beta$ coronaviruses, however, it seems Nsp1 is not highly conserved among the lineage of viruses $[5,6]$. Nsp1 has 180 amino acids (aa) forming two unstructured domains and one domain working as an interconnecting region between the two unstructured domains [7]. The crystal structure of SARS-CoV-2 Nsp1 revealed a six-stranded and capped $\beta$-barrel motif similar to SARS-CoV Nsp1 [8]. Based on previous studies, the interaction between the helical hairpin at the C-terminal domain of Nsp1 and the host 40S subunit of ribosome prohibits the entry of mRNA into the host ribosome [9]. Therefore, Nsp1 leads to translational shutoff of proteins from host mRNAs by binding the 40S ribosome of the host cell [9-11]. The C-terminal domain of Nsp1 has two helices [10] that are inserted into the entrance area of the ribosomal mRNA channel, probably contributing to the translational blockage $[9,10]$. In fact, Nsp1 leads to an endonucleolytic cleavage near the 5' UTR of host mRNA (capped mRNA) when binding to ribosome [12]. Moreover, Nsp1 inhibits all cellular antiviral defense mechanisms such as Type I interferon (IFN-I) response, which leads to the shutoff of the response of the innate immune system $[10,13]$. Nsp1 directly binds to SARS-CoV-2 stem-loop 1 (SL1) of 5-UTR RNA, which it seems this interaction can increase viral gene replication in the host cells by recruiting the host 40S ribosome at that region [5]. Examination of the physical properties of the SARS-CoV-2 Nsp1 has shown that has a positive surface charge [14]. Despite the existence of a structural model of the Nsp1 $[9,15]$, few studied have been conducted on the dynamic 
aspects of this protein and how mutations may be change its conformation and function. Here, we used molecular dynamic (MD) to further investigate the structure of Nsp1 in SARS-CoV-2 and SARS-CoV-1 and determined the effect of mutations on Nsp1 stability. These observations highlight the relevance of understanding how mutations effect the Nsp1 inhibition of immune response and binding to the ribosome.

\section{Computational Methods}

\subsection{Sequence and structure alignment}

The sequences of wild type (Polyprotein1ab) NSP1 proteins isolated from the SARS-CoV-2 and SARSCoV-1 viruses were retrieved from UniProtKB (http://www.uniprot.org/), under the identifier numbers of P0DTD1 and P0C6X7, respectively. To investigation of interaction between NSP1 and ribosome, structure of complex (PDB code: 7K5I) was downloaded from RCSB and a complete model of complex retrieved from SARS-CoV-2 3D (https://sars3d.com/). The automated sequence alignment was performed using the EMBOSS Needleman-Wunsch method $[16,17]$. This tool indicates the percentage of identity and similarity between both nsp1. I-TASSER server [18] was utilized to build the 3D structure of both NSP1, which is an online server for automated protein structure prediction. The best model was selected based on its C-score and the quality of the predicted structure was confirmed by PROCHECK $[19,20]$ and ProSA [21], which calculate an overall quality score for 3D structures. The local environment of amino acids was investigated through the WHAT IF coarse packing quality control, which should stay above 5 [22]. Mutations were also predicted with the use of the Dynamut mutation prediction. In the end, $\mathrm{H++}$ server was used to predict the protonation of histidine residues at $7.4 \mathrm{pH}$ [23]. UCSF Chimera (1.14) is a program that was used to visualization and analysis of structures [24].

\subsection{Nsp1 complexes, mutations and calculation of binding energy}

SARS-CoV-2 Nsp1 mutations (substitutions, insertions, and deletions) were retrieved from the CoV-GLUE database [25]. PRODIGY web server [26] was used to predict the binding affinity, number and type of intermolecular contacts within the $5.5 \AA$ distance cut off of Nsp1-ribosome complex from it 3D structure. The identified substitutions in the Nsp1 C-terminal (helix-turn-helix) were examined in the DynaMut [27] web server to determine their effects on protein stability. The effect of mutations in protein stability and the estimation of change in the folding free energy upon the mutation (DDG ${ }^{\text {Destablizing }}$ [ [27, 28] and vibrational entropy energy (DDS ${ }^{\mathrm{Vis}}$ ) was predicted using the DynaMut tool [27]. MutaBind2 [29] was used to evaluate the effects of mutations on Nsp1-ribosome interactions. This server computed changes in binding affinity upon single mutations (DDG ${ }^{\text {Binding }}$ ).

\subsection{Molecular dynamics simulation}

The molecular dynamics (MD) simulation were carried out using the GROMACS (2020.2). A water box was created with at least $1 \mathrm{~nm}(10 \AA)$ distances from the protein using the SPC water model and applying boundary conditions. The system neutralization was done by adding $\mathrm{Na}^{+}$and $\mathrm{Cl}^{-}$ions at the 
concentration of $0.1 \mathrm{M}$. The MD simulation was carried out to examine the quality of the model structures by investigating their stability via performing $100 \mathrm{~ns}$ simulations at a constant temperature $310 \mathrm{~K}$ (NVT). Energy minimization was performed in 50,000 steps to avoid any bad contacts generated while solvating the system. Then, the NPT optimization was done for 100 ps. To increase the likelihood of achieving the appropriate structure, the MD simulation was performed for $100 \mathrm{~ns}$ using the OPLS force field. The MD simulation was performed in three replicas for SARS-CoV-2 and two for SARS-CoV-1 Nsp1. In addition the MD simulation was carried out for mutant types of SARS-CoV-2 Nsp1. At the end of this process, the GROMACS Tools package were used for the trajectory analysis. In addition, CHARMM-GUI [30] was used to generate inputs of Nsp1-RNA complex for GROMACS and the MD simulation was carried out for $~ 73$ ns using CHARMM force field.

\subsection{Network analysis of Nsp1-ribosome complex and intrinsically disordered protein}

RING 2.0 [31] was used to represent 2D structure of Nsp1-ribosome complex and it was applied for the identification of covalent and non-covalent bonds between Nsp1 and ribosome subunits, including $\pi-\pi$ stacking, Hbond and VDW. The network visualized using Cytoscape. This network represents nodes (e.g. residues) and edges (e.g. bonds between the residue side chains), which are essential factors to network analysis.

Traditional structural studies have shown that proteins need a well-defined structure to perform their function. In contrast, recent structural studies have introduced proteins that have parts that do not form a specific three-dimensional structure and are known as Intrinsically disordered regions (IDRs) [3234]. Recognition of the natural frequency and functional importance of IDRs and ordered regions of proteins were identified as an essential factor in studying different biological processes [33]. PONDR ${ }^{\circledR}$ has been used to investigate IDRs of SARS-CoV-2 Nsp1, which is based on several different predictors such as VL3 and VSL2 $[35,36]$. In each of these predictors, the first letter refers to the method of characterization (V: Various) and the second letter refers to the length of the disordered region (S: Short (8 - 9 residues), and L: Long (40 or more residues)).

\section{Results}

\subsection{Sequence and structure alignment}

The results of the amino acid sequence alignment revealed that the Nsp1 of SARS-CoV-2 shares $84.4 \%$ identity and $91.1 \%$ similarity with the Nsp1 of SARS-CoV-1. The tertiary structure of SARS-CoV-2 NSP1 has consisted of regular secondary structural elements arranged sequentially as $\beta 1, \alpha 1, \beta 2, \alpha 2, \beta 3, \beta 4, \beta 5$, $\beta 6, a 3$, and $a 4$, which secondary structure is composed of a mixture of parallel and antiparallel $\beta$-strands

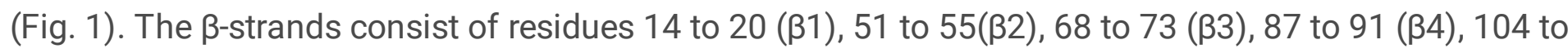
109 ( $\beta 6)$, and 117 to $123(\beta 6)$, while $\beta 5, \beta 1$ and $\beta 6$ are shorter and $\beta 3$ and $\beta 4$ are longer than identical $\beta$ strands of SARS-CoV-1 Nsp1. Also, NSP1 contains four a-helixes namely a1 (35 to 49), a2 (60 to 63), a3 (153 to 160), and a4 (164-178) that a3 and a4 locate at the C-terminal of protein. Investigation of the 
Nsp1-ribosomecomplex revealed that the a3 and a4 play a key role in forming this complex. In particular, several specific residues (Y154, E155, D156, F157, Q158, E159, N160, W161, T170, L173, M174, L177, $\mathrm{N} 178$ ) in this region were identified as essential residues involved in the interaction with components of the 40S ribosome subunit (U3 and S2). Most of these residues are found in the list of SARS-CoV-2 Nsp1 mutations. Therefore, examining important residues and understanding their position in protein sequence and structure can provide a novel insight into the effect of mutations on protein function. Furthermore, the Nsp1 has only one cysteine at position 51 , which is the first amino acid of $\beta 2$. This residue is significant because its neighboring residues may mutate and create an extra cysteine. It is suggested that it may lead to form disulfide bond and increase the stability of the protein structure.

\subsection{Analysis of Nsp1 complexes and effect of mutations}

The complex structure of the SARS-CoV-2 Nsp1 protein and human ribosomal 40S subunit were analyzed. The binding site of Nsp1 to the ribosome contains 26 residues at Nsp1 C-terminal. Among these residues, 17 residues form a helix-turn-helix $(\mathrm{HTH})$ motif and lead to form Nsp1-ribosome complex. $\mathrm{HTH}$ interacts simultaneously with different regions of the ribosome called "head region" including uS3 and "body region" including eS30 and uS5. HTH interacts with uS3 by D152, E155, D156, E159, Q158, and N160 residues, as well as this motif involved in interaction with uS5 by Y154, F157, Q158, W161, T170, L173, L177, N178 residues. R175, E176, and G179 residues of HTH motif play a key role in forming Nsp1-

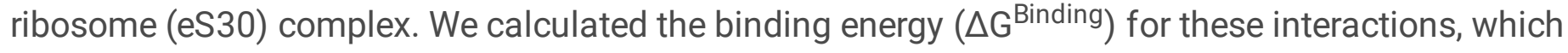
showed that the interaction between the Nsp1 and uS3 is stronger than the other interactions (Table 1).

Table 1

Binding affinity $(\Delta G)$ and dissociation constant $\left(K_{d}\right)$ predicted values for the interaction between SARS-CoV-2 Nsp1 and human ribosome.

\begin{tabular}{|lll|}
\hline Protein-protein complex & $\Delta G(\mathrm{kcal} / \mathrm{mol})$ & $\mathrm{K}_{\mathrm{d}}(\mathrm{M})$ at $\mathbf{3 7 . 0} \mathbb{C}$ \\
(Viral Nsp1/ribosome) & & \\
\hline Nsp1-uS3 & -6.9 & $1.3 \mathrm{E}-05$ \\
\hline Nsp1-uS5 & -5.6 & $1.1 \mathrm{E}-04$ \\
\hline Nsp1-eS30 & -4.1 & $1.2 \mathrm{E}-03$ \\
\hline
\end{tabular}

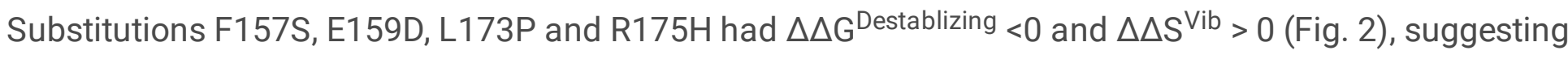
that they may increase the infectivity of host cells by destabilizing the Nsp1 and increasing its entropy and flexibility. Other mutations may have the opposite effect on Nsp1, such as E159K, T170I, M174K and $\mathrm{N} 178 \mathrm{~K}$, which have $\Delta \Delta \mathrm{G}^{\text {Destablizing }}>0$ and $\Delta \Delta \mathrm{S}^{\mathrm{Vib}}<0$, which stabilize the protein and reduce its entropy (Fig. 2). These substitutions may reduce the rate at which the Nsp1 binds to the host ribosomal subunits and thus reduces the rate of infection. 
We identified W161 in the HTH motif as causing a turn in that motif, leading to the helix disruption. This residue can also cause hydrophobic interactions (with F157) and stabilize the protein structure. Several mutations are known for this residue, including W161C, W161S, W161L, and W161R. We found that

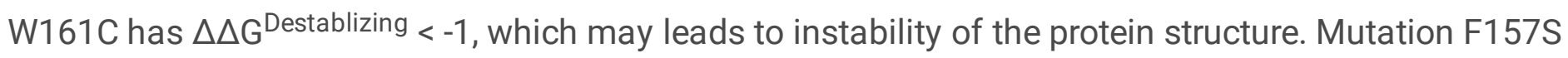
can also affect the formation or loss of this hydrophobic interaction, which in turn probably impacts the stability of the Nsp1 in that region.

Investigation of Nsp1-RNA complex have shown that protein can interact with viral RNA through their residues, which include 41E, 47K, 48D, 49G, 50T, 51C, 72K, 75D, 77R, 82G, 96Q, 97T, 99R, 102E, 112G, 113E and 114I (with Hydrogen bond)/ 122L (Hydrophobic interaction)/ 31F ( $\pi$-stacking) (Fig. 3a). Several residues at the surface interaction of Nsp1-RNA are known to have mutated. Among the most frequent mutations (Table 2), G112 change to $\mathrm{C} / \mathrm{S}$ and $\mathrm{G} 49$ to $\mathrm{C}$ result in $\Delta \Delta \mathrm{G}^{\text {Destabilizing }}$ below zero. These results suggested that the mutation of glycine to cysteine and serine could lead to protein instability, increased flexibility of Nsp1 and increase entropy of this protein. These factors can increase the rate at which Nsp1 binds to viral RNA. D75G and L122F mutations also showed negative values for $\Delta \Delta G^{\text {Destabilizing }}$ and $\Delta \Delta S^{V i s}$, indicating that they affect structure stability and thermodynamics parameters (i.e. decrease entropy and protein flexibility). A third group of mutations (D75E and E102K) leads to increase stability, decrease entropy and flexibility of protein, which perhaps reduce protein binding to the viral RNA, thereby reducing infectivity. The last group of mutations includes D48G, which showed positive values for $\Delta \Delta \mathrm{G}^{\text {Destabilizing }}$ and $\Delta \Delta \mathrm{S}^{\mathrm{Vis}}$. Based on results of calculations by computational tools, this mutation appears to increase stability and entropy of Nsp1.

Figure $3 b$ shows the surface potential of the Nsp1-RNA complex that indicating the Nsp1 binds to viral RNA through its positive residues in that region.

Some mutations also have effects on intra-atomic interactions of Nsp1 that lead to the elimination and formation of interactions such as van der Waals (yellow), weak Hydrogen bond (orange), and hydrophobic interactions (green), polar (light pink), as shown in Fig. 4. 
Table 2

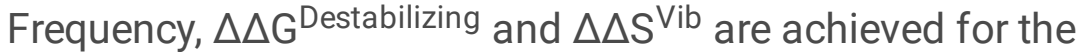
mutations of Nsp1 N-ter.

\begin{tabular}{|llll|}
\hline Mutation & Frequency & $\begin{array}{l}\Delta \Delta G^{\text {Destabilizing }} \\
(\mathrm{kcal} / \mathrm{mol})\end{array}$ & $\begin{array}{l}\Delta \Delta \mathbf{S}^{\mathrm{Vib}} \\
\left(\mathrm{kcal}^{\mathrm{m}} \mathrm{mol}^{-1} \cdot \mathrm{K}^{-1}\right)\end{array}$ \\
\hline G112C & 102 & -0.800 & 0.032 \\
\hline G49C & 118 & -1.765 & 0.084 \\
\hline G112S & 145 & -1.060 & 0.039 \\
\hline D75G & 161 & -0.244 & -0.005 \\
\hline L122F & 224 & -0.077 & -0.811 \\
\hline I114V & 232 & -0.097 & 0.058 \\
\hline E102K & 321 & 0.534 & -0.185 \\
\hline I114L & 403 & -0.164 & 0.072 \\
\hline D75E & 990 & 0.848 & -0.265 \\
\hline D48G & 1185 & 0.001 & 0.089 \\
\hline
\end{tabular}

\subsection{MD simulation}

Molecular dynamics (MD) simulations were performed for the wild type and mutant types of SARS-CoV-2 Nsp1 and SARS-CoV-1 Nsp1. The RMSD plot indicates that all proteins reached equilibrium after $100 \mathrm{~ns}$ and that the SARS-CoV-1 Nsp1 deviation is less than the SARS-CoV-2 Nsp1 (Fig. 5a). We identified four main deletions in Nsp1 at sites 80-90, 156-158, 158-161 and 175-177. The deletions result in different conformations in Nsp1 C-terminal region (Fig. 6). SARS-CoV-2 Nsp1 ${ }^{(\Delta 175-177)}$ and Nsp1 ${ }^{(\Delta 1158-161)}$ follow a pattern similar to SARS-CoV-1 Nsp1 and have less fluctuations than other proteins. This result indicates that the Nsp1 has a lower value of RMSD with the 175-177 and 158-161 deletions, so the mutated proteins are more stable than the wild protein and other mutant protein (Fig. 5a). In contrast, $\Delta 156-158$ and $\triangle 80-90$ have higher RMSD values than SARS-CoV-2 Nsp1 wild type, which may indicate increasing protein fluctuations and instability.

The SARS-CoV-1 Nsp1 exhibited a lower $\mathrm{R}_{\mathrm{g}}$ values compared to the SARS-CoV-2 Nsp1 (Fig. 5b). The difference between $\mathrm{R}_{\mathrm{g}}$ of these two proteins is $\sim 0.12 \mathrm{~nm}$. Overall, all models of SARS-CoV-2 Nsp1 had various values of $\mathrm{Rg}$ that including Nsp1 ${ }^{(\Delta 156-158)}>\mathrm{Nsp} 1^{\text {(wild) }}>\mathrm{Nsp} 1^{(\Delta 80-90)}>\mathrm{Nsp} 1^{(\Delta 175-177)}>\mathrm{Nsp} 1$ $(\Delta 158-161)$. Nsp1 ${ }^{(\Delta 156-158)}$ has more functional movements due to its higher value of $R_{\mathrm{g}}$ and lesser compactness. 
The RMS plot was constructed from the 100 ns data in order to understand the deviation of each Nsp1 (Fig. 5c). The RMSF of the backbone for the SARS-CoV-2 Nsp1 displayed more flexible 164 to 170 residues (Lys, His, Ser, Ser, Gly, Val, and Ter), as compared to the SARS-CoV-1 Nsp1. This region corresponds to the C-terminal of the Nsp1 related to the protein interaction with the ribosome through CT, meaning that its flexibility could be related to its function. As shown in Fig. $5 \mathrm{c}$, the RMSF plot of mutant proteins had more fluctuations compared to wild Nsp1 due to their deleted residues. In order to understand the total motion of Nsp1 structures in the phase space, the first two eigenvectors were projected onto it. The results indicate that the motion features characterized by the two eigenvectors are different in the SARS-CoV-2 and SARS-CoV-1 Nsp1 proteins. SARS-CoV-2 Nsp1 show wider motions than SARS-CoV-1 Nsp1, indicating more conformational changes.

As shown in Fig. 5(d-e), the values of RMSD and $\mathrm{R}_{\mathrm{g}}$ resulting from the analysis of Nsp1-RNA trajectories after $73 \mathrm{~ns} \mathrm{MD}$ simulation are $0.6 \mathrm{~nm}$ and $1.5 \mathrm{~nm}$, respectively. The RMSF plot of this complex also showed fluctuations in the site of RNA binding to the protein (Fig. 5f).

\subsection{Analysis of Network and IDRs of Nsp1}

Critical residues at the interface between the SARS-CoV-2 Nsp1 and ribosome subunits in their complex are indicated in the RIN presented (Fig. 7). Key residues were determined by using stress and betweenness as the two main parameters of the local metrics. High betweenness values represent that the node is a mediator of interactions with other nodes and that is probably a key structural residue, while stress is defined as the number of shortest paths passing through a node. The analysis of NSP1 (Cterminal) network revealed crucial residues including F157, V169, T170, and L173. These amino acids were identified as critical residues at the interface between the SARS-CoV-2 NSP1 and ribosome. Furthermore, these residues were known as amino acids that have mutated.

In addition, Table 3 describes the most important residues of the SARS-CoV-2 involved in the interaction between its Nsp1 and ribosome via $\mathrm{H}$-bonds, van der Waals bonds, and $\pi-\pi$ stacking interactions. Among these residues, $\Delta 158-161, \Delta 156-158, \Delta 175-177, F 157 S, E 159 D, L 173 P$ and R175H are critical because their mutations could alter the ability of the Nsp1 to bind to ribosome, which could lead to the loss of efficacy of drugs targeting the Nsp1 or competing with it for ribosome. 
Table 3

Representation of interaction between different residues of the SARS-CoV-2 Nsp1 and ribosome subunits.

\begin{tabular}{|c|c|c|}
\hline Residues of Ribosome & Residues of Nsp1 & Interaction \\
\hline (uS5) & 178ASN & VDW \\
\hline 105GLU & 177LEU & VDW \\
\hline 106VAL & 157PHE & VDW \\
\hline 109ILE & 173LEU & VDW \\
\hline 109ILE & 161TRP & VDW \\
\hline 122THR & 161TRP & PIPISTACK \\
\hline 124PHE & 161TRP & VDW \\
\hline 124PHE & 154TYR & VDW \\
\hline 147VAL & 158GLN & HBOND \\
\hline 147VAL & 158GLN & VDW \\
\hline 147VAL & 161TRP & VDW \\
\hline 147VAL & 177LEU & VDW \\
\hline \multicolumn{3}{|l|}{ 151ILE } \\
\hline (uS3) & 155GLU & HBOND \\
\hline 115VAL & 152ASP & HBOND \\
\hline 116ARG & 155GLU & HBOND \\
\hline 116ARG & 155GLU & VDW \\
\hline 116ARG & 155GLU & HBOND \\
\hline 143ARG & 156ASP & VDW \\
\hline 143ARG & 159GLU & VDW \\
\hline \multicolumn{3}{|l|}{ 143ARG } \\
\hline (eS30) & 175ARG & VDW \\
\hline 52LYS & & \\
\hline
\end{tabular}

The VSL2 predictor was used to characterize disordered regions of any length and identified accurately the short disordered regions of SARS-CoV-2 NSP1 at 120-180 positions (Fig. 8). PONDR VL3 predictor was applied to determine SARS-CoV-2 NSP1 tendency for disorder. This predictor identified regions of interest at positions 150-180 (Fig. 8). The regions identified as disorder regions are approximately 
related to the $\mathrm{C}$-terminal protein. It is also suggested that the occurrence of mutations in this region may increase these disorders in the protein. Structural studies revealed that IDRs of virus structure influence viral genome (RNA) adaptive capacity, host-virus interactions, virus-host range, cross-species transmission, and host tropism [37-41]. In antigen selection, IDRs are significantly considered because these disordered regions may induce an undesirable immune response (weak or even completely nonimmune responses)[42].

\section{Discussion}

The COVID-19 epidemic is spreading as an unsolvable problem worldwide. Despite the efforts of researchers, many details on SARS-CoV-2 biology remain unknown. Our study focused on the Nsp1 structure and stability by using different computational tools. We found that SARS-CoV-2 Nsp1 has a reduced compactness in comparison with SARS-CoV-1 Nsp1. In addition, we found that the C-terminal of the SARS-CoV-2 Nsp1 is more flexible than other regions of SARS-CoV-2 Nsp1 and SARS-CoV-1 Nsp1, confirming the role of this region in the interaction with the $40 \mathrm{~S}$ subunit.

Interestingly, a previous study suggested that when SARS-CoV-2 5'-UTR bind to Nsp1 N-terminal, the covalently linked Nsp1 C-terminal is not able to bind the 40S subunit [43]. The authors suggest that this may be due to a steric factor in which Nsp1 C-terminal is unable to approach the $40 \mathrm{~S}$ subunit to form the Nsp1-ribosome complex. Several mutations in the Nsp1 N-terminal may change the affinity of Nsp1 binding to the 5'-UTR of SARS-CoV-2, perhaps making it more effective in using the host ribosome for translation of viral proteins.

Clinical data showed that D500-532 in Nsp1 N-terminal correlated with lower viral load and lower serum IFN- $\beta$ that indicating its clinical significance [44]. Therefore, mutations can have a major effect on pathogenicity of SARS-CoV-2. According to the results of MD simulation analysis, it can be suggested that mutations, especially deletion mutations, can play a vital role in protein structure and function. For example, based on MD analysis, D156-158 maybe increase the rate of binding of Nsp1 to ribosome subunit and mutations D158-161 and D175-177 may affect protein binding to the ribosome and reduce pathogenicity of the virus. Moreover, substitution mutations such as M174K/T170I mutations and E159D, R175H, L173P and F157S in the HLH motif of Nsp1 C-terminal could alter the thermodynamics parameters that may affect the interactions of the Nsp1-ribosome complex and rate of infectivity of SARS-CoV-2. Hence, investigation the results of in silico studies and comparing them with experimental data can show the association of mutations with the immune system response as well as the function of the virus in the host cell.

\section{Conclusion}

Among 795 Nsp1 substitutions, 69 belonged to the HTH motif in the C-terminal region. In this article, out of 69 mutations, only 11 substitutions with a frequency above 10 were examined and it was revealed that these mutations affect the thermodynamic parameters of the interaction between the protein and the 
ribosome. The effect of HTH mutations may increase the pathogenicity of SARS-CoV-2 and in turn, increase the inhibition of host protein translation. Important mutations were identified as important mutations in the Nsp1 N-terminal that may affect the affinity binding of viral RNA to this protein. Therefore, findings suggest that Nsp1 should be considered as an important virulence factor because more mutations in the binding motif of Nsp1 may have drastic consequences.

\section{Declarations}

\section{Acknowledgements}

The support and resources from the Center for High Performance Computing at Shahid Beheshti University of Iran are gratefully acknowledged. We would also like to thank all the researchers who have kindly shared genomes on public databases.

\section{Funding}

This research has been conducted using the research credits of Shahid Beheshti University, G.C., Tehran, Iran (Grant number; SAD/600/1451). This work was partially supported by the Fundação para a Ciência e a Tecnologia [RESEARCH 4 COVID-19 project n. 029] and the EOSCsecretariat.eu COVID-19 Fast Track Funding. EOSCsecretariat.eu has received funding from the European Union's Horizon Programme call H2020-INFRAEOSC-05-2018-2019, grant Agreement number 831644.

\section{References}

1. Gordon DE, Jang GM, Bouhaddou M et al (2020) A SARS-CoV-2 protein interaction map reveals targets for drug repurposing. Nature 583:459-468. https://doi.org/10.1038/s41586-020-2286-9

2. Clark LK, Green TJ, Petit CM (2020) Structure of Nonstructural Protein 1 from SARS-CoV-2. J Virol 95:. https://doi.org/10.1128/jvi.02019-20

3. Egorova T, Alkalaeva E (2020) Nsp1 of SARS-CoV-2. Stimulates Host Translation Termination

4. Kamitani W, Narayanan K, Huang C et al (2006) Severe acute respiratory syndrome coronavirus nsp1 protein suppresses host gene expression by promoting host mRNA degradation. Proc Natl Acad Sci U S A 103:12885-12890. https://doi.org/10.1073/pnas.0603144103

5. Vankadari N, Jeyasankar NN, Lopes WJ (2020) Structure of the SARS-CoV-2 Nsp1/5'-Untranslated Region Complex and Implications for Potential Therapeutic Targets, a Vaccine, and Virulence. J Phys Chem Lett 9659-9668. https://doi.org/10.1021/acs.jpclett.0c02818

6. Tohya Y, Narayanan K, Kamitani W et al (2009) Suppression of Host Gene Expression by nsp1 Proteins of Group 2 Bat Coronaviruses. J Virol 83:5282-5288. https://doi.org/10.1128/jvi.02485-08

7. Pandala N, Cole CA, McFarland D et al (2020) A Preliminary Investigation in the Molecular Basis of Host Shutoff Mechanism in SARS-CoV. Proc 11th ACM Int Conf Bioinformatics, Comput Biol Heal 
Informatics, BCB 2020. https://doi.org/10.1145/3388440.3412483

8. Semper C, Watanabe N, Savchenko A (2021) Structural characterization of nonstructural protein 1 from SARS-CoV-2. iScience 24:101903. https://doi.org/10.1016/j.isci.2020.101903

9. Schubert K, Karousis ED, Jomaa A et al (2020) SARS-CoV-2 Nsp1 binds the ribosomal mRNA channel to inhibit translation. Nat Struct Mol Biol 27:959-966. https://doi.org/10.1038/s41594-020-0511-8

10. Thoms M, Buschauer R, Ameismeier M et al (2020) Structural basis for translational shutdown and immune evasion by the Nsp1 protein of SARS-CoV-2. Science 369:1249-1256. https://doi.org/10.1126/SCIENCE.ABC8665

11. Lokugamage KG, Narayanan K, Huang C, Makino S (2012) Severe Acute Respiratory Syndrome Coronavirus Protein nsp1 Is a Novel Eukaryotic Translation Inhibitor That Represses Multiple Steps of Translation Initiation. J Virol 86:13598-13608. https://doi.org/10.1128/jvi.01958-12

12. Tanaka T, Kamitani W, DeDiego ML et al (2012) Severe Acute Respiratory Syndrome Coronavirus nsp1 Facilitates Efficient Propagation in Cells through a Specific Translational Shutoff of Host mRNA. J Virol 86:11128-11137. https://doi.org/10.1128/jvi.01700-12

13. Bouayad A (2020) Innate immune evasion by SARS-CoV-2: Comparison with SARS-CoV. Rev Med Virol 30:1-9. https://doi.org/10.1002/rmv.2135

14. Vankadari N, Jeyasankar NN, Lopes WJ (2020) Structure of the SARS-CoV-2 Nsp1/5'-Untranslated Region Complex and Implications for Potential Therapeutic Targets, a Vaccine, and Virulence. J Phys Chem Lett 11:9659-9668. https://doi.org/10.1021/acs.jpclett.0c02818

15. Almeida MS, Johnson MA, Herrmann T et al (2007) Novel $\beta$-Barrel Fold in the Nuclear Magnetic Resonance Structure of the Replicase Nonstructural Protein 1 from the Severe Acute Respiratory Syndrome Coronavirus. J Virol 81:3151-3161. https://doi.org/10.1128/jvi.01939-06

16. Needleman SB, Wunsch CD (1970) A general method applicable to the search for similarities in the amino acid sequence of two proteins. J Mol Biol 48:443-453. https://doi.org/10.1016/00222836(70)90057-4

17. Rice P, Longden L, Bleasby A (2000) EMBOSS: The European Molecular Biology Open Software Suite. Trends Genet 16:276-277. https://doi.org/10.1016/S0168-9525(00)02024-2

18. Yang J, Zhang Y (2015) I-TASSER server: New development for protein structure and function predictions. Nucleic Acids Res 43:W174-W181. https://doi.org/10.1093/nar/gkv342

19. Laskowski RA, MacArthur MW, Moss DS, Thornton JM (1993) PROCHECK: a program to check the stereochemical quality of protein structures. J Appl Crystallogr 26:283-291. https://doi.org/10.1107/s0021889892009944

20. Laskowski RA, Rullmann JAC, MacArthur MW et al (1996) AQUA and PROCHECK-NMR: Programs for checking the quality of protein structures solved by NMR. J Biomol NMR 8:477-486. https://doi.org/10.1007/BF00228148

21. Wiederstein M, Sippl MJ (2007) ProSA-web: Interactive web service for the recognition of errors in three-dimensional structures of proteins. Nucleic Acids Res 35:407-410. https://doi.org/10.1093/nar/gkm290 
22. Vriend G, Sander C (1993) Quality control of protein models: Directional atomic contact analysis. J Appl Crystallogr 26:47-60. https://doi.org/10.1107/S0021889892008240

23. Anandakrishnan R, Aguilar B, Onufriev AV (2012) H + + 3.0: Automating pK prediction and the preparation of biomolecular structures for atomistic molecular modeling and simulations. Nucleic Acids Res 40:537-541. https://doi.org/10.1093/nar/gks375

24. Pettersen EF, Goddard TD, Huang CC et al (2004) UCSF Chimera - A visualization system for exploratory research and analysis. J Comput Chem 25:1605-1612. https://doi.org/10.1002/jcc.20084

25. Singer J, Gifford R, Cotten M, Robertson D (2020) CoV-GLUE: A Web Application for Tracking SARSCoV-2 Genomic Variation. Preprints 2020060225. https://doi.org/10.20944/preprints202006.0225.v1

26. Xue LC, Rodrigues JP, Kastritis PL et al (2016) PRODIGY: A web server for predicting the binding affinity of protein-protein complexes. Bioinformatics 32:3676-3678. https://doi.org/10.1093/bioinformatics/btw514

27. Rodrigues CHM, Pires DEV, Ascher DB (2018) DynaMut: predicting the impact of mutations on protein conformation. flexibility stability 46:350-355. https://doi.org/10.1093/nar/gky300

28. Rezaei S, Sefidbakht Y, Uskoković V (2020) Comparative molecular dynamics study of the receptorbinding domains in SARS-CoV-2 and SARS- CoV and the effects of mutations on the binding affinity. J Biomol Struct Dyn 0:1-20. https://doi.org/10.1080/07391102.2020.1860829

29. Zhang N, Chen Y, Lu H et al (2020) MutaBind2: Predicting the Impacts of Single and Multiple Mutations on Protein-Protein Interactions. iScience 23:100939. https://doi.org/10.1016/j.isci.2020.100939

30. Allouche A (2012) Software News and Updates Gabedit - A Graphical User Interface for Computational Chemistry Softwares. J Comput Chem 32:174-182. https://doi.org/10.1002/jcc

31. Piovesan D, Minervini G, Tosatto SCE (2016) The RING 2. 0 web server for high quality residue interaction networks. 44:367-374. https://doi.org/10.1093/nar/gkw315

32. Lee RV, Der, Buljan M, Lang B et al (2014) Classi fi cation of. Intrinsically Disordered Regions and Proteins

33. Uversky VN (2019) Intrinsically disordered proteins and their "Mysterious" (meta)physics. Front Phys 7:8-23. https://doi.org/10.3389/fphy.2019.00010

34. Vacic V, Markwick PRL, Oldfield CJ et al (2012) Disease-Associated Mutations Disrupt Functionally Important Regions of Intrinsic Protein Disorder. PLoS Comput Biol 8:. https://doi.org/10.1371/journal.pcbi.1002709

35. Lieutaud P (2016) How disordered is my protein and what is its disorder for ? A guide through the " dark side" of the protein universe. 4:1-33. https://doi.org/10.1080/21690707.2016.1259708

36. Vinterhalter G, Kova JJ, Uversky VN, Pavlovi GM (2021) International Journal of Biological Macromolecules Bioinformatics analysis of correlation between protein function and intrinsic disorder. 167:446-456. https://doi.org/10.1016/j.ijbiomac.2020.11.211 
37. Charon J, Barra A, Walter J et al (2018) First Experimental Assessment of Protein Intrinsic Disorder Involvement in an RNA Virus Natural Adaptive Process. Mol Biol Evol 35:38-49. https://doi.org/10.1093/molbev/msx249

38. Walter J, Charon J, Hu Y et al (2019) Comparative analysis of mutational robustness of the intrinsically disordered viral protein VPg and of its interactor elF4E. PLoS One 14:1-13. https://doi.org/10.1371/journal.pone.0211725

39. Mozzi A, Forni D, Cagliani R et al (2020) Intrinsically disordered regions are abundant in simplexvirus proteomes and display signatures of positive selection. Virus Evol 6:1-12. https://doi.org/10.1093/ve/veaa028

40. Barik S (2020) Genus-specific pattern of intrinsically disordered central regions in the nucleocapsid protein of coronaviruses. Comput Struct Biotechnol J 18:1884-1890. https://doi.org/10.1016/j.csbj.2020.07.005

41. Sen S, Dey A, Bandhyopadhyay S, Uversky VN (2012) Understanding Structural Malleability of the SARS-CoV-2 Proteins and their Relation to the Comorbidities SARS-CoV-2. 774:1-17

42. Macraild CA, Richards JS, Anders RF, Norton RS (2016) Antibody Recognition of Disordered Antigens. Structure 24:148-157. https://doi.org/10.1016/j.str.2015.10.028

43. Shi M, Wang L, Fontana P et al (2020) SARS-CoV-2 Nsp1 suppresses host but not viral translation through a bipartite mechanism. bioRxiv 2:1-16. https://doi.org/10.1101/2020.09.18.302901

44. Lin J, Tang C, Wei H, cheng et al (2021) Genomic monitoring of SARS-CoV-2 uncovers an Nsp1 deletion variant that modulates type I interferon response. Cell Host Microbe 29:489-502.e8. https://doi.org/10.1016/j.chom.2021.01.015

\section{Figures}




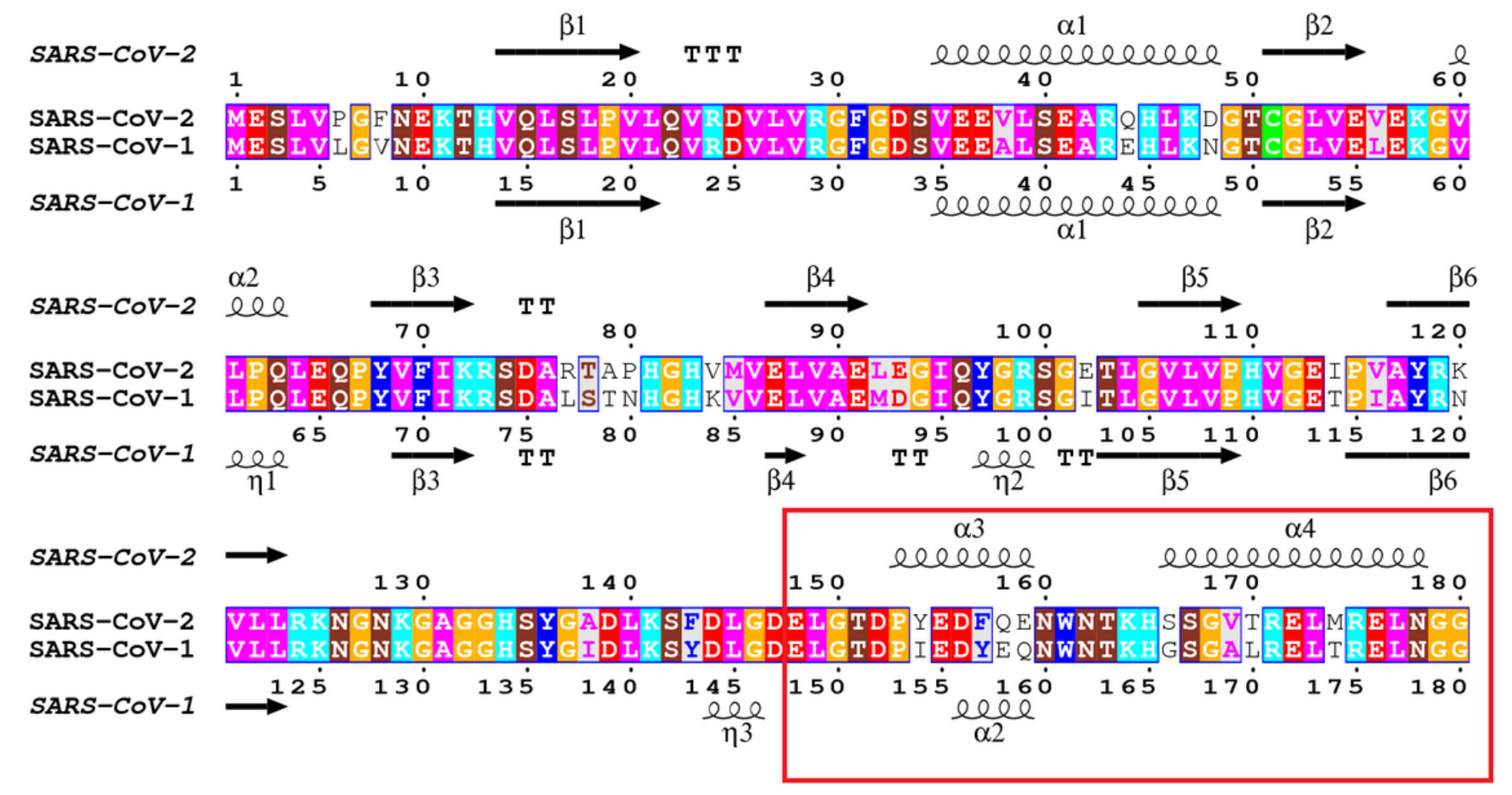

Figure 1

Structure and sequence alignment between SARS-CoV-2 NSP1 and SARS-CoV-1 NSP1; Red box indicates the region that interacts with the ribosome. 


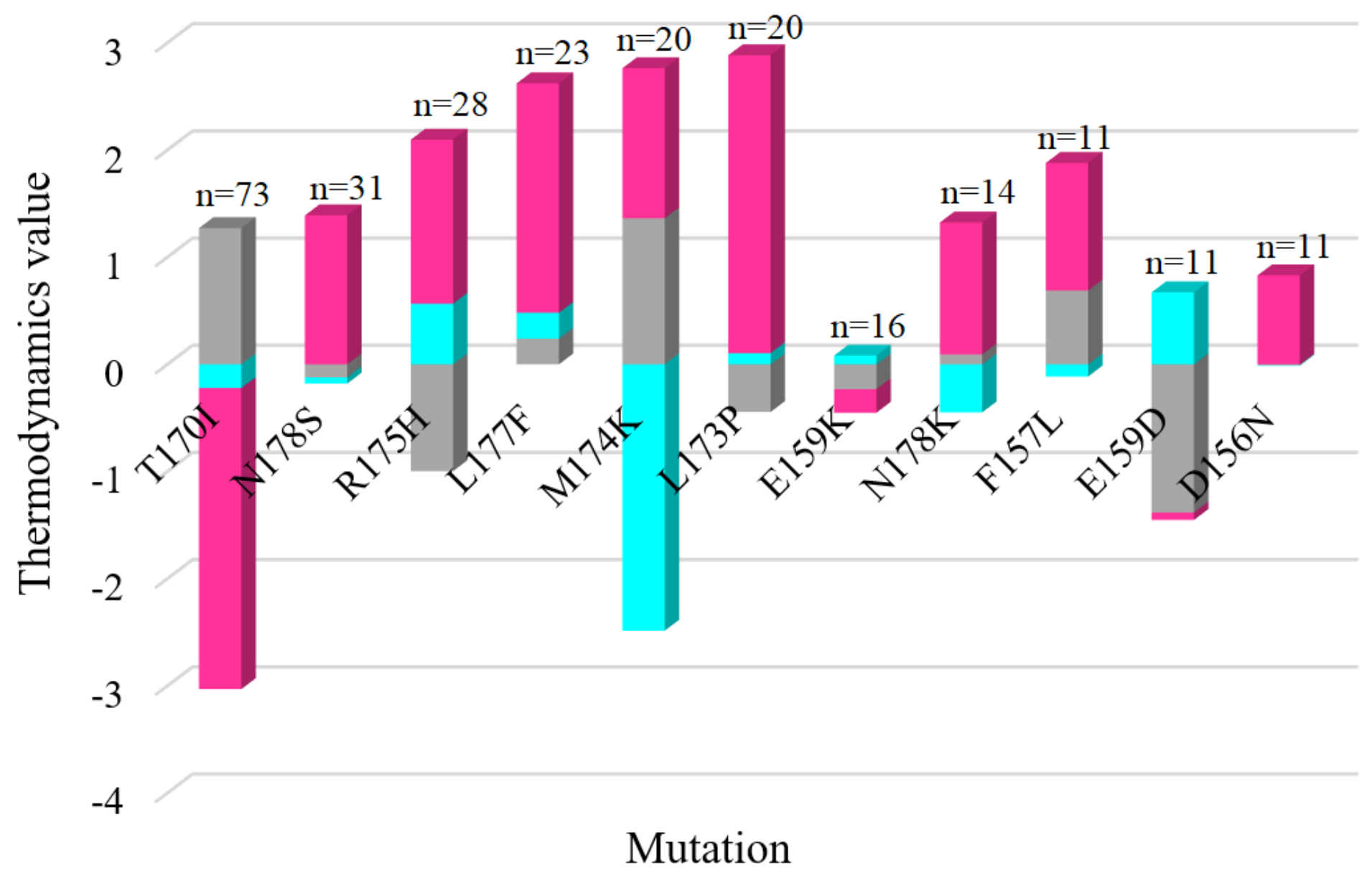

$\Delta \Delta \mathrm{G}^{\text {Destabilizing }}-\Delta \Delta \mathrm{S}^{\mathrm{Vib}}=\Delta \Delta \mathrm{G}^{\text {binding }}$

Figure 2

Representation of effect of Nsp1 (HTH motif) mutations on thermodynamics parameters including $\square$ GDestabilizing, $\Delta \Delta S V i b$, and $\square$ Gbinding . 

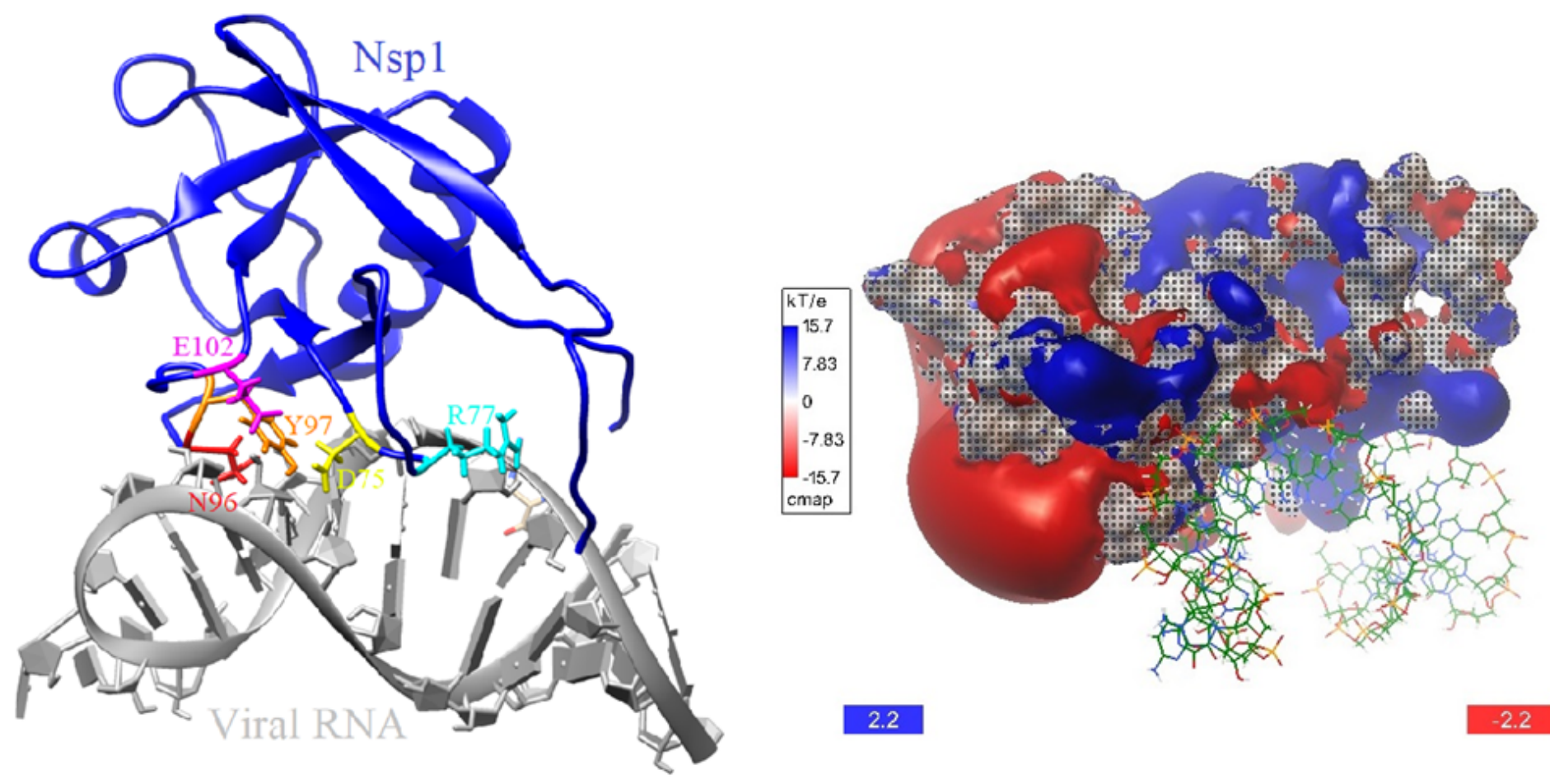

Figure 3

(a) RNA-protein complex and RNA-binding residues and (b) Surface potential of Nsp1-RNA complex are represented.

(a)
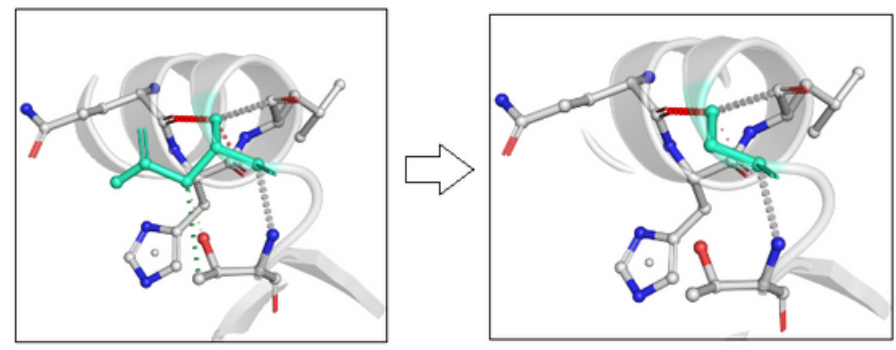

(c)
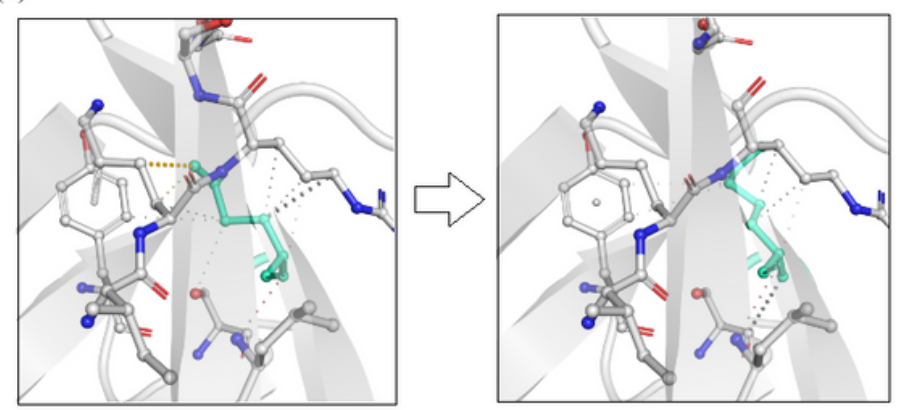

(b)

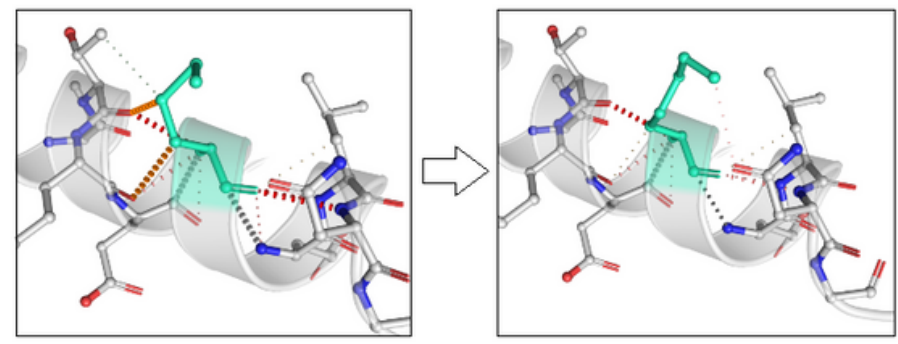

(d)

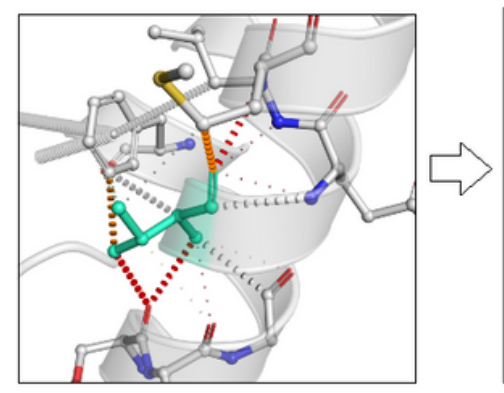

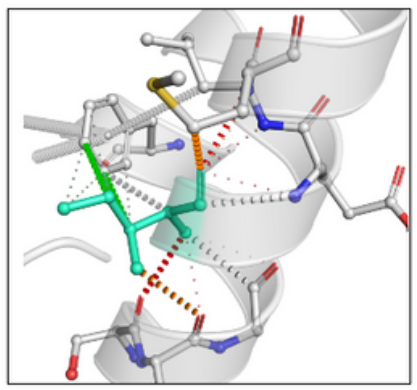

Figure 4 
Effect of mutations on interatomic interactions of Nsp1. (a) D48G, (b) E102K, (c) M174K and (d) T170I.
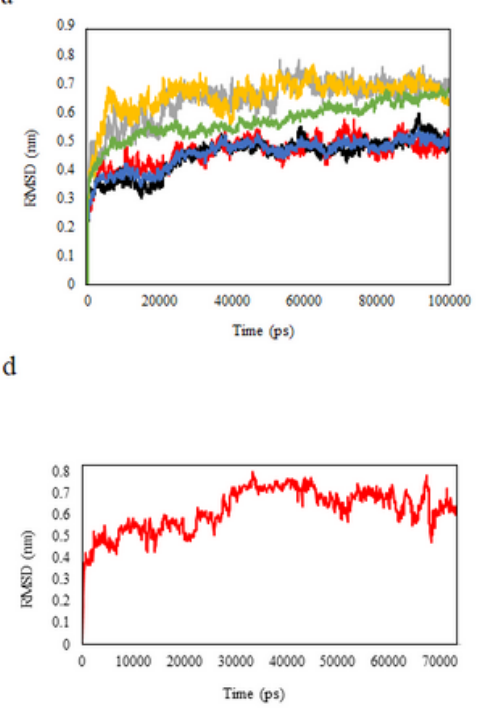
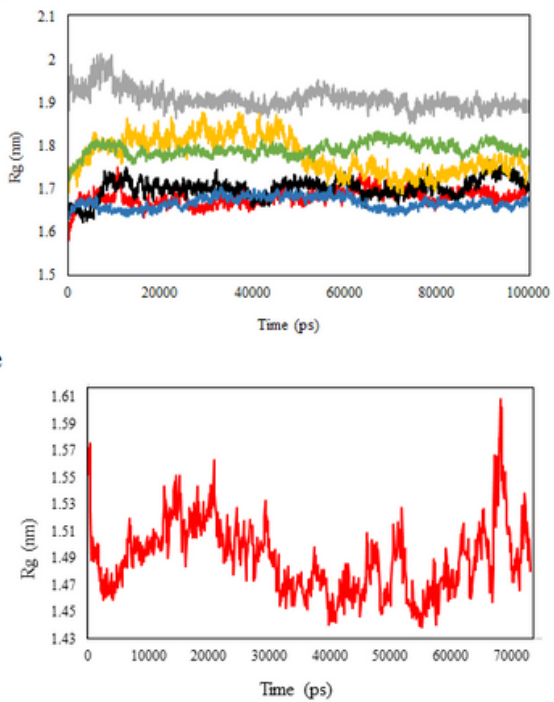

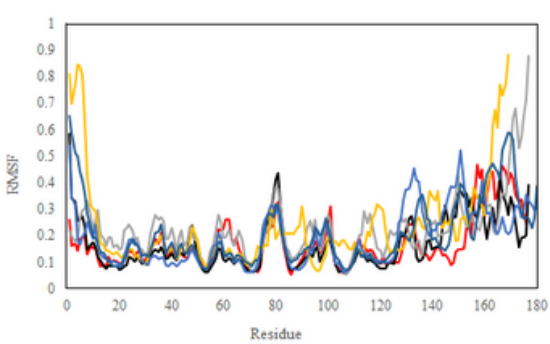

f

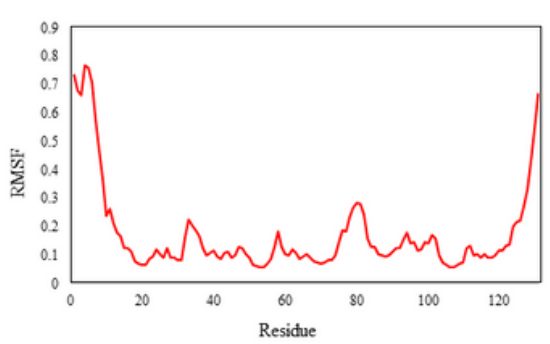

- Mutant (del 158-161)

- Mutant (del 175-177)

- Mutant (del 156-158)

- Mutant (del 80-90)

- SARS-CoV-1 Nspl

- SARS-CoV-2 Nsp1

Figure 5

MD simulation analysis. (a)RMSD, (b) Rg, and (c) RMSF of the SARS-CoV-2 and SARS-CoV-1 NSP. (d-f) Analysis of Nsp1-RNA complex.

(a)

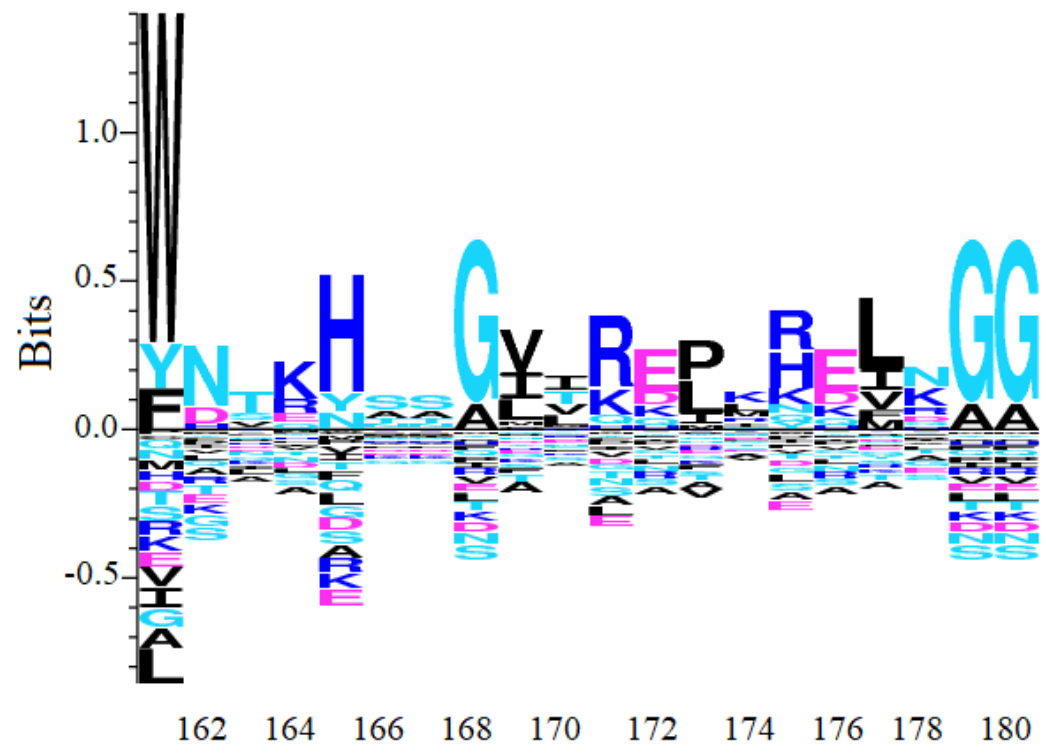

(b)

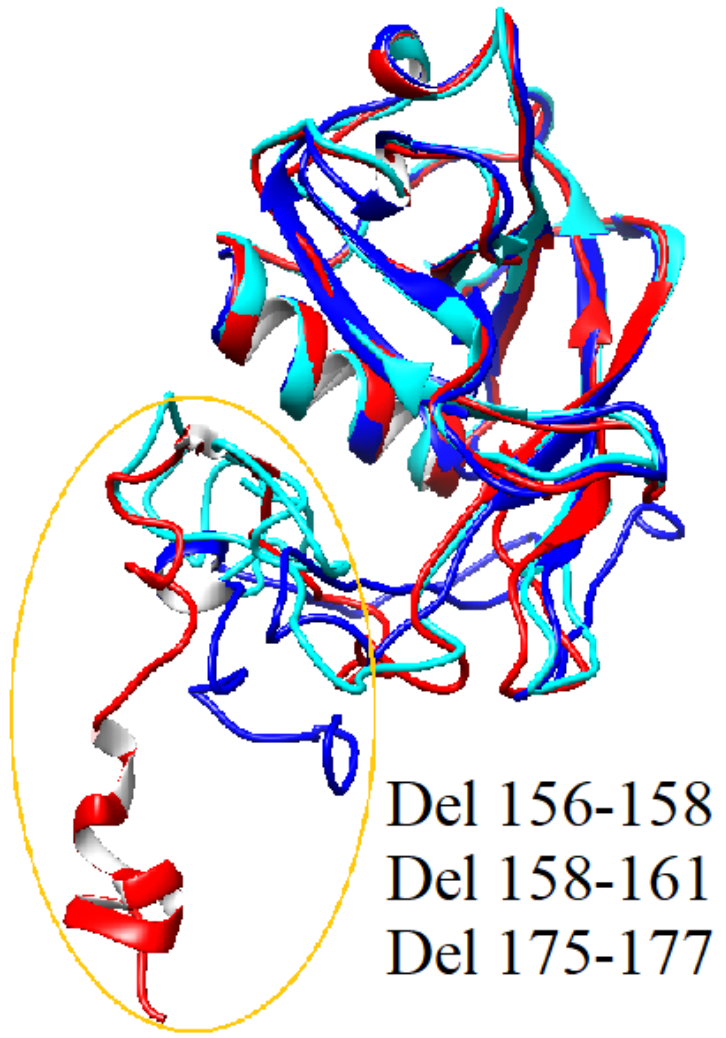

Figure 6 
(a) Sequence logo of SARS-CoV-2 Nsp1 C-terminal (160-180 residues), and (b) representation of superimposed structures of Nsp1 mutants that indicate regions including deletions.

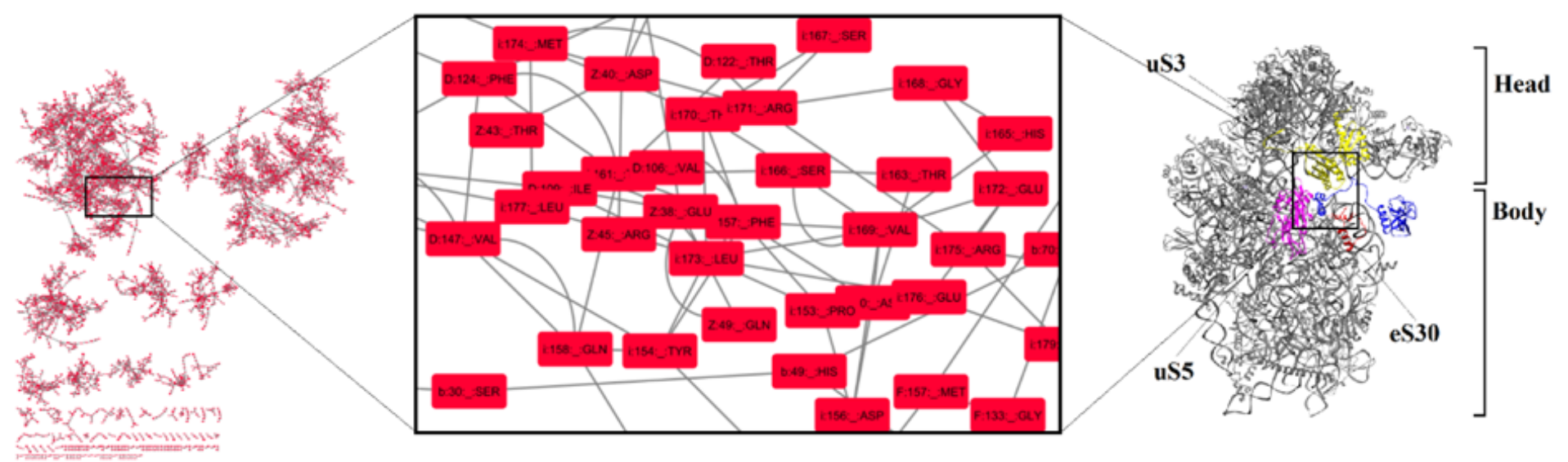

Figure 7

2D and 3D representation of Nsp1-ribosome complex and interface interaction between viral protein and human ribosome.

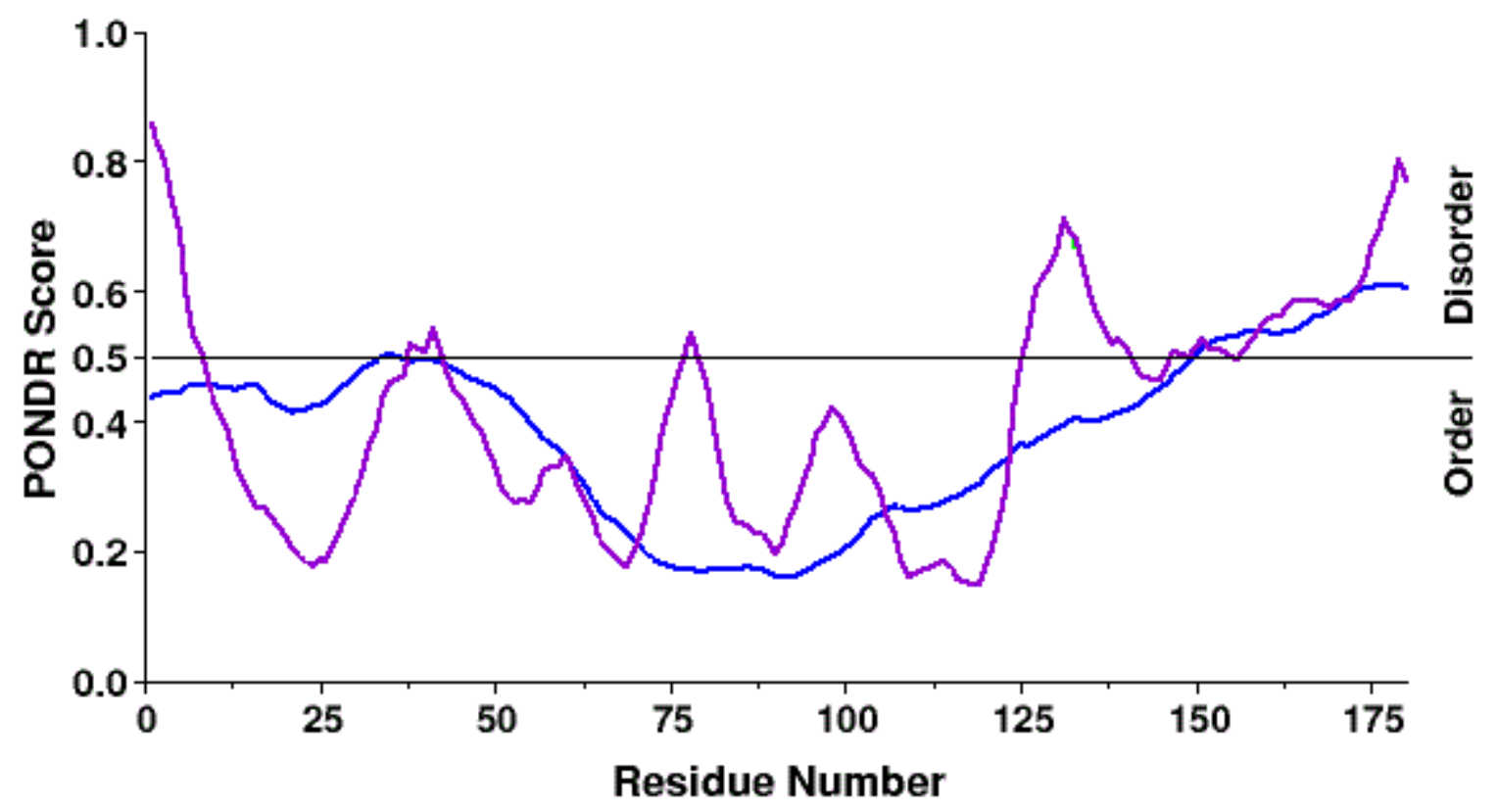

Figure 8

Representation of IDRs of SARS-CoV-2 NSP1 by using PONDR.

\section{Supplementary Files}


This is a list of supplementary files associated with this preprint. Click to download.

- GraphicalAbstract.png 